\title{
ITERATED LOOP FUNCTORS AND THE HOMOLOGY OF THE STEENROD ALGEBRA
}

BY WILLIAM M. SINGER ${ }^{1}$

Communicated by S. Eilenberg, November 3, 1975

Let $A$ be the mod-2 Steenrod algebra. For any unstable $A$-module $M$ the "unstable homology groups" $H_{s, k}^{A}(M)=\operatorname{Tor}_{s, k}^{A}(M)$ are defined by means of unstable projective resolutions of $M[2]$. We describe here a new approach to the problem of computing these groups.

Let $M_{A}$ be the category whose objects are unstable $A$-modules and whose morphisms are degree preserving $A$-maps. For $M$ in $M_{A}$ and $x$ in $M_{n}$ we write, as is usual $\mathrm{Sq}_{a} x=\mathrm{Sq}^{n-a} x$. Let "suspension" $S: M_{A} \rightarrow M_{A}$ be the functor that raises degree by 1 . $S$ has a left adjoint $\Omega: M_{A} \rightarrow M_{A}$ [2] given by $(\Omega M)_{n}=$ (coker $\left.\mathrm{Sq}_{0}\right)_{n+1}$, with $A$-action induced by that on $M$. The left derived functors $\Omega_{s}(s \geqslant 0)$ of $\Omega$ are defined in the usual way: given $M$ in $M_{A}$ one forms a projective resolution $\cdots \rightarrow P_{1}(M) \rightarrow P_{0}(M) \rightarrow M \rightarrow 0$. Then $\Omega_{s} M$ is the $s$ th homology group of the complex $\cdots \rightarrow \Omega P_{1}(M) \rightarrow \Omega P_{0}(M) \rightarrow 0$. The left derived functors of $\Omega$ are completely understood [1], [2], [3]. In fact,

$$
\begin{gathered}
\Omega_{s} M=0 \quad \text { if } s>1, \\
\left(\Omega_{1} M\right)_{2 n-1}=(\operatorname{ker~Sq})_{n}
\end{gathered}
$$

with $A$-action given by $\mathrm{Sq}_{a} \Omega_{1} x=\Omega_{1} \mathrm{Sq}_{(a+1) / 2}$ for $x$ in ker $\mathrm{Sq}_{0}$.

Consider now the $k$-fold iterate $\Omega^{k}$ of $\Omega$. We pose:

PRoblem (*). Give a workable description of the left derived functors $\Omega_{s}^{k}$ of $\Omega^{k}$, for all $s \geqslant 0$.

Our interest in these derived functors stems from the fact that their zerodimensional components are the unstable homology groups of the Steenrod algebra:

THEOREM 1. There is a natural isomorphism $\operatorname{Tor}_{s, k}^{A}(M)=\left(\Omega_{s}^{k} M\right)_{0}$.

Our interest in Problem (*) is heightened by the fact that it appears to be solvable: there is a simple relation between the derived functors of $\Omega^{k}$ and those of $\Omega^{k-1}$.

AMS (MOS) subject classifications (1970). Primary 55G10, 55H15; Secondary 18G10, $18 \mathrm{H} 15$.

1 Research partially supported by NSF grant MPS 75-08278.

Copyright $\odot$ 1976, American Mathematical Society 
THEOREM 2. There is a natural long exact sequence of $Z_{2}$-modules:

$$
\begin{aligned}
\cdots \rightarrow \Omega_{s}^{k-1} M & \stackrel{\mathrm{Sq}_{0}}{\longrightarrow} \Omega_{s}^{k-1} M \rightarrow \Omega_{s}^{k} M-\cdots \\
& \rightarrow \Omega_{s-1}^{k-1} M \stackrel{\mathrm{Sq}_{0}}{\longrightarrow} \Omega_{s-1}^{k-1} M \rightarrow \cdots
\end{aligned}
$$

and consequently a short exact sequence in $M_{A}$ :

$$
0 \rightarrow \Omega \Omega_{s}^{k-1} M \rightarrow \Omega_{s}^{k} M \rightarrow \Omega_{1} \Omega_{s-1}^{k-1} M \rightarrow 0 .
$$

This result seems to promise a quick inductive description of the functors $\Omega_{s}^{k}$; however, computation of examples with small values of $k$ and $s$ show that the short exact sequence (4) is in general not split over $M_{A}$ !

Our main result (Theorem 3 below) is the construction for each unstable $A$-module $M$ of a small chain complex $L^{k} M=\Sigma_{s} L_{s}^{k} M$ from which the derived functions $\Omega_{s}^{k} M$ can be computed: $H_{s}\left(L^{k} M\right)=\Omega_{s}^{k} M$. We seek, in particular, complexes that can be fit into a short exact sequence:

$$
0 \rightarrow L^{k-1} M \stackrel{\alpha}{\rightarrow} L^{k} M \stackrel{\beta}{\rightarrow} L^{k-1} M \longrightarrow 0
$$

for which the associated long exact sequence in homology is the same as (3). This consideration motivates us in our definition of the graded $Z_{2}$-module $L_{s}^{k} M$ $=\Sigma_{n \geqslant 0}\left(L_{s}^{k} M\right)_{n}$ : we set $\left(L_{0}^{k} M\right)_{n}=M_{n+k}$, and proceed inductively by setting $L_{s}^{k} M=\Sigma_{i=0}^{k-1}\left(L_{s-1}^{i} M\right)$, defining dimension by

$$
\operatorname{dim}\left(0,0, \ldots, x^{i}, \ldots, 0\right)=2 \operatorname{dim} x^{i}-(k-i) \text { for } x^{i} \text { in } L_{s-1}^{i} .
$$

Then $\alpha$ in (5) is just the inclusion of $\sum_{i=0}^{k-2}\left(L_{s-1}^{i} M\right)$ into $\sum_{i=0}^{k-1}\left(L_{s-1}^{i} M\right)$, while $\beta$ is just the projection of $\Sigma_{i=0}^{k-1}\left(L_{s-1}^{i} M\right)$ onto $L_{s-1}^{k-1} M$. If we ignore grading, $L_{s}^{k} M$ is just the direct sum of $\left(\begin{array}{l}k \\ s\end{array}\right)$ copies of $M$. Our main result is

TheOREM 3. For all $k \geqslant 0, s \geqslant 0, a \geqslant 0$ there are natural $Z_{2}$-homomorphisms $d_{s}: L_{s}^{k} M \rightarrow L_{s-1}^{k} M, \lambda_{s}(a): L_{s}^{k} M \rightarrow L_{s}^{k} M$ with the following properties:

(a) $d_{s-1} d_{s}=0$ so that $L^{k} M$ is a chain complex. $\alpha, \beta$ in (5) are chain maps.

(b) The operators $\lambda_{s}(a)$ satisfy Adem relations "up to homotopy": if $b>$ a there are $Z_{2}$-linear maps $\beta_{s}(b, a): L_{s}^{k} M \rightarrow L_{s+1}^{k} M$ such that

$$
\begin{aligned}
\lambda_{s}(b) \lambda_{s}(a)-\sum_{j \leqslant b / 2}\left(\begin{array}{c}
j-1 \\
a-b+2 j
\end{array}\right) & \lambda_{s}(b-2 j) \lambda_{s}(a+j) \\
& =d_{s+1} \beta_{s}(b, a)+\beta_{s-1}(b, a) d_{s} .
\end{aligned}
$$

(c) $d_{s} \lambda_{s}(a)=\lambda_{s-1}(a) d_{s}$, so that $\lambda_{s}(a)$ can be regarded as an operator on $H_{s}\left(L^{k} M\right)$.

(d) The operations $\lambda_{s}(a)$ vanish on $H_{s}\left(L^{k} M\right)$ if $a<k$, and $H_{s}\left(L^{k} M\right)$ becomes an unstable A-module if we put $\mathrm{Sq}_{a}=\lambda_{s}(a+k)$ for all $a \geqslant 0$. 
(e) There is a natural isomorphism of unstable a-modules $H_{s}\left(L^{k} M\right)=\Omega_{s}^{k} M$, and the long exact sequence in homology associated with (5) is identical with (3).

Details of this construction and applications to the computation of $\Omega_{s}^{k} M$ will appear elsewhere. We mention only that if $S^{n}$ is the unique $M_{A}$ object for which $\left(S^{n}\right)_{n}=Z_{2},\left(S^{n}\right)_{j}=0$ if $j \neq n$, then the differential $d_{s}: L_{s}^{k} S^{n} \rightarrow L_{s-1}^{k} S^{n}$ vanishes if $k \leqslant n+s-1$. This fact permits us to determine completely the unstable $A$-modules $\Omega_{s}^{k} S^{n}$ for those cases in which $k \leqslant n+s-1$. For example, it turns out that $\Omega_{s}^{n+s^{-1}} S^{n}$ is the suspension of a truncated polynomial algebra over $A$ of a kind already classified by Sugawara and Toda in [4].

\section{BIBLIOGRAPHY}

1. A. K. Bousfield and E. B. Curtis, A spectral sequence for the homotopy of nice spaces, Trans. Amer. Math. Soc. 151 (1970), 457-479. MR 42 \#2488.

2. W. S. Massey and F. P. Peterson, The mod 2 cohomology structure of certain fiber spaces, Mem. Amer. Math. Soc. No. 74 (1967). MR 37 \#226. $367-382$.

3. W. M. Singer, The algebraic EHP sequence, Trans. Amer. Math. Soc. 201 (1975),

4. T. Sugawara and H. Toda, Squaring operations on truncated polynomial algebras, Japan. J. Math. 38 (1969), 39-50. MR 41 \#4530.

DEPARTMENT OF MATHEMATICS, FORDHAM UNIVERSITY, BRONX, NEW YORK 10458 